\title{
Autopsy and Medicolegal Evaluation in a Case of Sudden Maternal Death from Pulmonary Embolism
}

\author{
Pawan Mittal ${ }^{1}$, Prateek Karagwal', Meenu Gilotra ${ }^{3}$, Amrita Kulhria $^{4}$, Abhishek Saini ${ }^{5}$ and Saurabh \\ Juneja ${ }^{6}$
}

${ }^{1}$ Department of Forensic Medicine, Bhagat Phool Singh Government Medical College and Hospital for Women, Haryana, India ${ }^{2} 3^{r d}$ Year PG Resident, Department of Forensic Medicine, BPS GMC(W) Khanpur kalan, Haryana, India

${ }^{3}$ Medical Officer and Pathologist, District Civil Hospital, Haryana, India

${ }^{4}$ Assistant Professor, Shri Atal Bihari Vajpayee Government Medical College (SABVMC), India

${ }^{5} 3^{\text {rd }}$ Year PG Resident, Department of Pathlogy, BPS GMC(W) Khanpur kalan, Haryana, India

${ }^{6}$ Senior Resident, Department of Blood Transfusion, BPS GMC(W) Khanpur kalan, Haryana, India

*Corresponding author: Pawan Mittal, Department of Forensic Medicine, Bhagat Phool Singh Government Medical College and Hospital for Women (BPS GMC(W)), Khanpur Kalan, Sonepat, C-95, Sector 35, Suncity Township-I, Rohtak-124001, Haryana, India, Tel: 99-9198-6390

\begin{abstract}
Pregnancy and the postpartum period carry high risk of thromboembolic phenomenon which increases manifold postpartally. Multiple predisposing risk factors, usually acting in combination, add up to this risk. All three of the Virchow's triad conditions predisposing to vascular thrombosis, i.e., endothelial injury, venous stasis and hypercoagulability, are present in pregnancy. The deaths caused by pulmonary embolism may become the subject of medicolegal investigation due a sudden and unexpected fatal outcome. A thorough dissection, documentation and ancillary investigations pertaining to pulmonary emboli are important components of evaluating such fatalities. The investigation requires a dependable protocol that should include a proper evaluation of the timing of the embolus along with the residual and other thrombi, whenever discovered. Because of the pathophysiology and propagation of thrombus over a range of time period, one may see a broad histological range of thrombosis and organization. The present case pertains to the sudden death of a young female in the postpartum period who suffered a massive pulmonary embolism about 6 weeks after delivery. An extensive dissection of the lower limbs showed multiple deep venous thrombi in the calf and femoral regions while moderate to large coiled emboli were found in the pulmonary arterial vasculature. An additional histopathological dating of the thrombi as well as pulmonary embolus was performed that helped in elucidating the time range of their development.
\end{abstract}

\author{
Keywords \\ Pulmonary embolism, Vein, Pregnancy, Puerperium, \\ Dissection, Autopsy
}

\section{Introduction}

Venous thromboembolism (VTE) encompasses pulmonary emboli (PE) and deep venous thrombosis (DVT), typically of the pelvic and lower-extremity veins. The incidences of pulmonary embolism and DVT are difficult to calculate and are commonly underdiagnosed [1]. The mortality rate of an acute untreated $\mathrm{PE}$ is $30-35 \%$ [2]. Virchow described three risk factors for thrombosis, which bear his eponym 'Virchow's Triad'. These include endothelial injury, vascular stasis, and hypercoagulability [3].

Females during pregnancy and in postpartum period are particularly at the higher risk of thromboembolic phenomenon. These risks are due to the normal or physiological changes of pregnancy to save the blood loss of parturition [4]. However, the hemodynamic disturbances may also lead to the pathological changes of Virchow's triad. Pregnancy related pulmonary

Citation: Mittal P, Karagwal P, Gilotra M, Kulhria A, Saini A, et al. (2021) Autopsy and Medicolegal Evaluation in a Case of Sudden Maternal Death from Pulmonary Embolism. Int J Pathol Clin Res 7:121. doi. org/10.23937/2469-5807/1510121

Accepted: November 16, 2021: Published: November 18, 2021

Copyright: (C) 2021 Mittal P, et al. This is an open-access article distributed under the terms of the Creative Commons Attribution License, which permits unrestricted use, distribution, and reproduction in any medium, provided the original author and source are credited. 
embolism accounts for about $10 \%$ of maternal deaths throughout the world [5]. The incidence of pulmonary thromboembolism (PTE) is 10 times more common in the pregnant than the non-pregnant females in the same age group while the risk increases manifolds in the postpartum period which remains increased until approximately 12 weeks after delivery $[5,6]$.

The sudden and unexpected nature of death from fatal pulmonary thromboemblism may become the issue of medico-legal concern and a forensic pathologist is then expected to perform a thorough autopsy with ancillary investigations as well as appropriate evaluation of the circumstances surrounding death [7]. In cases of sudden deaths from PTE, the necessary autopsy protocol involves: (a) Demonstration of the pulmonary emboli and a search for the residual and additional thrombi by detailed dissection, esp. of the lower limbs and pelvic region, (b) Evaluation of the probable duration of thromboembolus with the help of histological methods, (c) A sound evaluation of the underlying etiology and, (d) The manner of death, i.e., natural versus non-natural.

\section{Case Report}

\section{Brief history}

The present case relates to a 21-years-old female, gravida one, married since one year and a village resident who delivered her first child (male) about 6 weeks back by lower segment caesarian section. The child was healthy with a full term and uneventful delivery. The female was obese with a significant lack of ambulation or physical activity, mostly spending a lethargic routine, as narrated by the attendants. She had complaints of relapsing and remitting pain and discomfort in legs since last one week which was not given any particular attention. On one evening while she got up from the bed and went to the toilet, she had a sudden onset and escalation of symptoms in the form of abdominal discomfort, chest pain and dyspnea and was taken to the local primary health centre (PHC) where her general status was found to be poor and the case was referred to the tertiary care centre. During transportation, she became unconscious after about an hour of onset of symptoms along with a single episode of seizure and did not respond thereafter. On arrival at the referral centre, she was declared 'dead on arrival'. The dead body was brought for autopsy after about 4 hours of death.

\section{External examination}

The female was apparently obese with a body weight of $95 \mathrm{~kg}$ and $165 \mathrm{~cm}$ in length. The clothes did not reveal any abnormal stains or odor. Postmortem hypostasis was livid purplish over dorsum of the body, face and root of the neck and blanching on blunt digital pressure. Rigor mortis was well developed on head, neck and upper limbs and re-established after breaking. No external injury or any other finding suspicious of foul play was present.

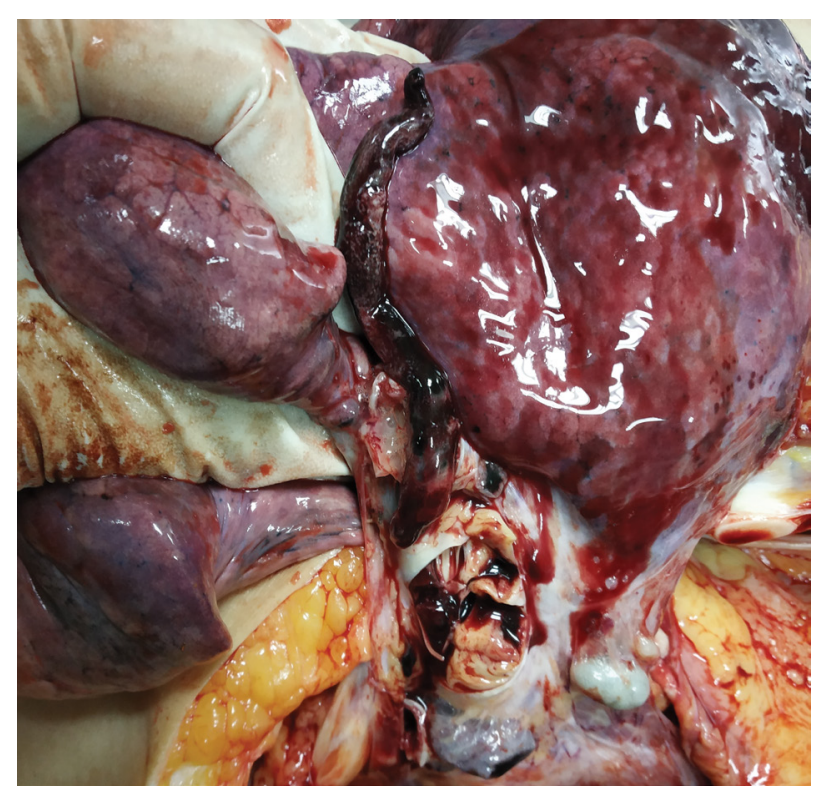

Figure 1: Long snake like embolus emerging out of right main pulmonary artery. Tan white to pinkish deposits over the clot may be seen (Zahn's Lines).

\section{Internal examination}

Gross findings: No evidence of any internal injury was present. A suspicion of a fatal pulmonary embolism was already evident during autopsy from the history and circumstances of the case which became obvious during removal of the lungs Dissection of the each lung at the hilum lead to the extrusion of moderate to large snakelike emboli from the main pulmonary arteries that were extending into further divisions (Figure 1). A large coiled embolus was found entangled within the musculature of right ventricle, as well as in the pulmonary trunk. The emboli further revealed a tan-white granular to pink layered deposit over the dark purplish clot. This differential pattern was further evident in different layers of the sectioned clot thereby defining Lines of Zahn, confirming their true ante mortem nature.

Following evisceration of thoracic, abdominal and cranial organs, the body was laid prone and lower limbs were dissected in search for deep venous thrombi. Serial sections and slight milking of each calf muscle extruded out paste-like and erect dark purplish thrombi from multiple veins while many of them were firmly adherent to the vascular walls (Figure 2). Upon proximal tracing and longitudinal opening, similar thrombi were found in femoral veins. Common iliac vein also depicted few adherent thrombi at random intervals. The viscera depicted changes of hypoxia and shock in the form of pallor and swelling with firm, pale and shiny cut surfaces.

Sections of the calf muscles containing vascular thrombi as well as the pulmonary emboli were preserved for detailed histopathological examination including their probable durations (Figure 3).

Histolopathological examination: Microscopic examination (H\&E) of the thrombus's section taken 


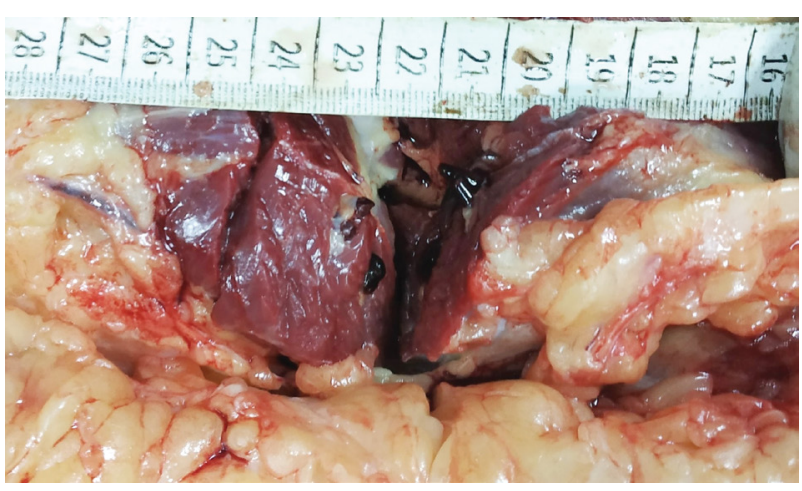

Figure 2: Sectioned right calf muscle depicting paste like and firm thrombi protruding out of the veins.

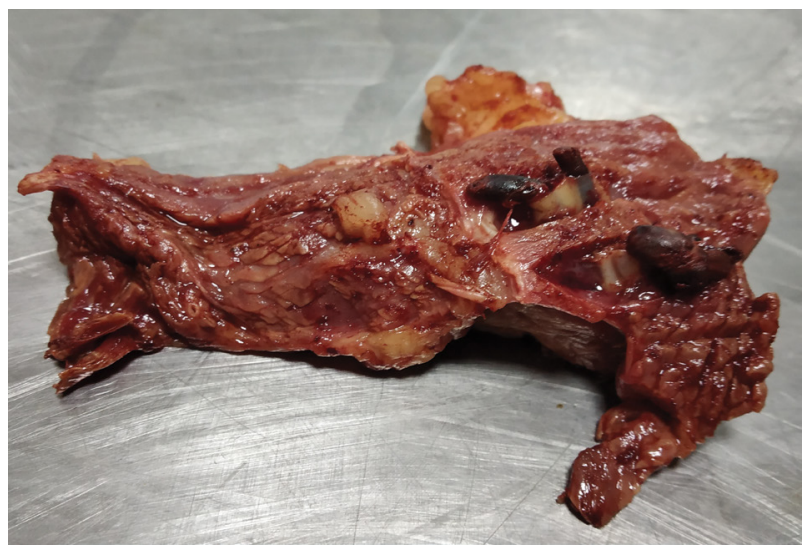

Figure 3: Calf muscle section along with contained thrombi preserved for histopathlogical evaluation.

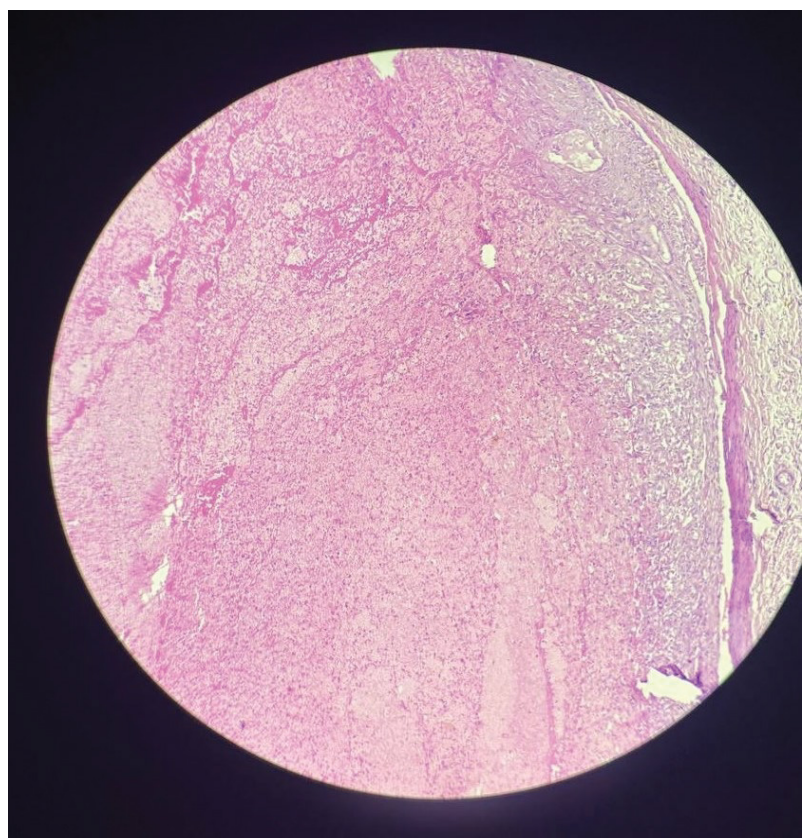

Figure 4: $H$ \& $E$ stained section from the popliteal vein thrombus revealing organization, with RBCs located centrally while histiocytes and fibroblasts are in the periphery (100x magnification).

from popliteal, femoral and pelvic veins resulted in different findings, varying from the acute changes of fibrin, RBCs and inflammatory cells' accumulations to

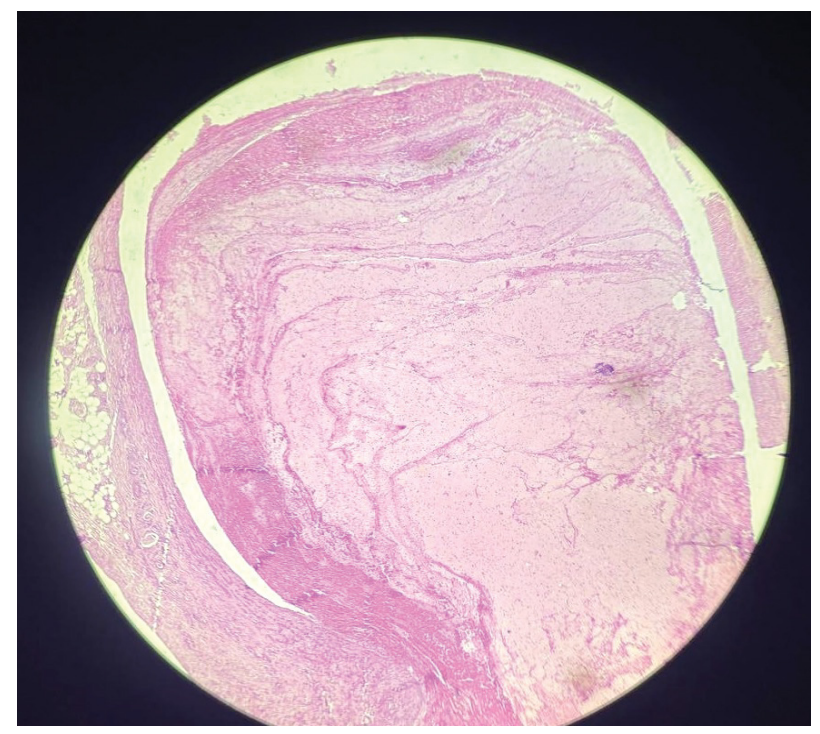

Figure 5: $H$ \& $E$ stained section from the femoral vein thrombus with abundant fibrin, RBCs and interspersed WBCs (100x magnification).

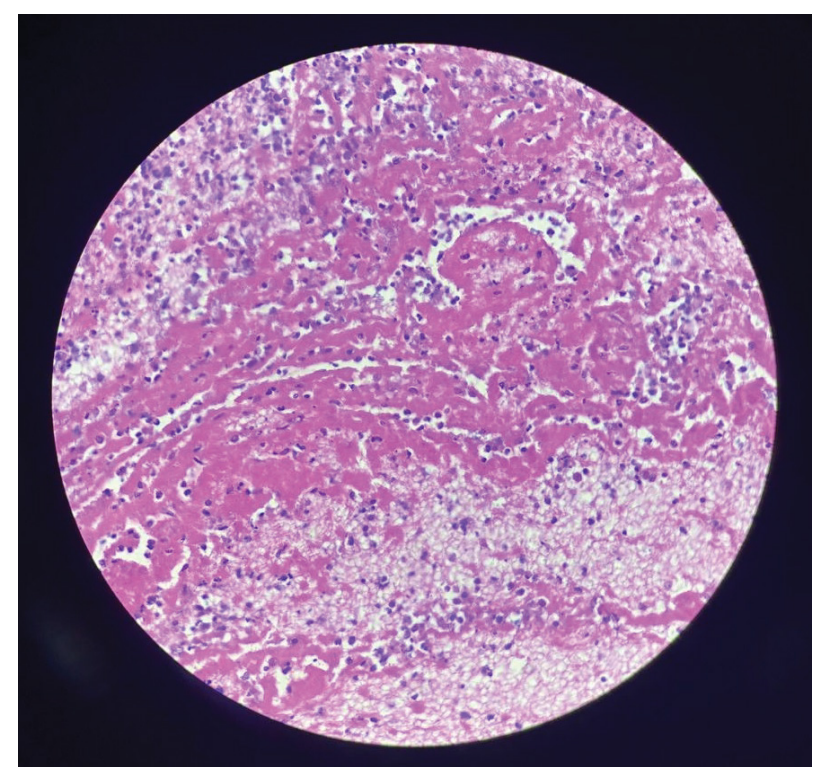

Figure 6: $H$ \& $E$ section from the most proximal pelvic thrombus revealing fibrin and interspersed inflammatory cells (400x magnification).

peripheral organization in the form of fibrosis (Figure 4, Figure 5 and Figure 6). There was an apparent distal (popliteal) to proximal (femoral-pelvic) progression of the histological changes from an organizing to acute thrombi respectively. The pulmonary embolus revealed histological changes similar to femoral and pelvic vein thrombi, i.e., accumulation of fibrin, erythrocytes and leucocytes. Thereby, the proximally extended femoropelvic thrombi were the most likely source of pulmonary embolism in the present case.

\section{Opinion and conclusion}

Toxicology results of viscera and blood sample were negative. The cause of death was given as acute pulmonary embolism due to deep venous thrombosis of 
the lower limb veins, leading to hypoxia and acute right heart failure. The manner of death was natural.

\section{Discussion}

Pulmonary embolism is the most under-diagnosed cause of death where no autopsy is usually performed and it has been estimated that less than half of fatal pulmonary emboli are recognized clinically [8]. In clinical pathology, postoperative pulmonary artery embolism and embolism associated with clinically manifest leg and/or pelvic deep vein thrombosis are predominant. Pregnancy associated PE occurs suddenly and is not easy to diagnose promptly as the most common symptom of $P E$ is dyspnea which may be the presentation of normal pregnancy $[9,10]$. The concurrence of typical symptoms and signs, i.e., dyspnea, chest pain and hemoptysis occurs in less than $25 \%$ of cases [11]. Forensic pathologists, however, also frequently see acute fatalities from PE without any previous symptoms of disease in seemingly healthy persons who die during routine activities. Usually, the deep veins of one or both legs still contain residual thrombi; the pelvic veins alone are rarely the origin of acutely lethal PE.

\section{Pregnancy and pulmonary artery thromboembo- lism (PTE)}

PTE is the leading cause of maternal mortality all over the world $[4,12]$. A woman's likelihood of venous thromboses is increased manifolds during pregnancy $[5,6]$, due to the conditions satisfying Virchow's triad. Vascular trauma occurs to some extent during both vaginal and operative delivery. Venous stasis occurs during pregnancy because the venous system dilates, and blood flow in the leg and pelvic veins is slowed by compression by the gravid uterus [13]. This may be augmented by immobility associated with bed rest. A state of hypercoagulability is induced by increases in certain clotting factors (including fibrinogen and factors II, VII, VIII, and X) and decreases or variable changes in some components of the fibrinolytic and anticoagulant systems (factor XI and others) $[14,15]$.

This increased coagulability is particularly significant if the woman has an underlying mutation in the prothrombin or factor $\mathrm{V}$ Leiden genes [16].

The risk of pulmonary artery thromboembolism is increased bothintheantepartumand postpartum periods [6]. Overall, pregnancy initiates a hypercoagulable state that spans a period of approximately 10 to 11 months [13]. The state of hypercoagulation is only temporary, and usually normalizes approximately 2 months after delivery [14].

The overall risk of venous thromboembolism in pregnancy is estimated at 1:1000 to 1:2000 [17]. The risk of PTE is considerably increased in the postpartum period and is several times common following a cesarean section delivery than a vaginal delivery $[17,18]$.
Risk factors of pregnancy associated with pulmonary embolism are history of previous surgery, preeclampsia, obesity, immobility, anemia, multiparity, age greater than 35 years and previous history of pregnancy associated pulmonary embolism [9].

\section{Consequences of the deep venous thrombi}

A thrombus may form in the deep leg veins because of endothelial injury, stasis, and/or a hypercoagulable state [3]. There are three possible fates of the thrombus: lysis, organization with recanalization and resorption, or embolism. Before resolution, the thrombus may extend further in the veins. Proximal propagation is the most dangerous because the risk of embolism is greatest from thrombi in the inguinal/proximal leg veins [19]. Because of the pathophysiology of propagation, this proximal thrombus is the most recent and therefore least adherent to the vessel wall. In fact, treatment of a DVT with anticoagulation therapy is aimed to stop the progression of the thrombus to allow time for the body to naturally lyse or organize and resorb the thrombus [20].

Once the thrombus dislodges and travels to the lung, the embolism, depending on its size and coexisting cardiovascular diseases, interferes with hemodynamics [21]. Increased pulmonary vascular resistance will cause right heart strain or failure. Inadequate gas exchange occurs because of decreased perfusion, which results in increased dead space and ventilation-perfusion mismatches. This, combined with decreased cardiac output, leads to global hypoxia [22].

Larger emboli wedge in the main pulmonary artery, whereas smaller emboli occlude the peripheral arteries and may cause pulmonary infarction(s).

\section{Dissection and search for the residual/source thrombus}

It may not be possible to always detect residual thrombus but dissection of the leg veins usually provides important information [23]. The chances of thrombus detection further depend upon the site and thoroughness of dissection and the technique(s) employed $[1,7]$.

The vast majority of pulmonary emboli arise from the venous thrombi situated in lower extremities and pelvis; however, other locations exist as well. Indwelling central venous catheters, upper extremity veins, and the right heart have been described as possible locations of thrombi [24]. An even more rare source is from jugular vein thrombosis, sometimes seen as an extension of intracranial dural venous sinus thrombosis (commonly superior sagittal sinus) that may also occur in the females in peripartum period, causing cerebral infarction and stroke $[1,4,12]$.

Axillary and subclavian vein thrombosis is equally unusual while the legs account for the vast majority of 
emboli. The origin of an embolus from the deep leg vein is usually suggested by its gross morphology, i.e., shape, diameter, and length.

\section{Histological dating of the thrombus}

The age assessment of a DVT carries special medicolegal significance. Various elaborative histological techniques, including special immunhistochemical methods, have been described to estimate the age of a thrombus [25-28]. Overall, one may determine whether a thrombus is acute, organizing, or remote. Because of the individual variability, it is often not possible to determine the precise age of a thrombus.

The histological appearance, however, can be compared and correlated with the clinical information for a corroborative evaluation. An opinion may be given on whether the age of a thrombus is consistent with a known history or not.

Once the thrombus forms, it can gradually enlarge as more fibrin-platelets are deposited and arranged in alternate layers with the erythrocytes, the so-called "Zahn's Lines" [26]. Zahn's lines are depictive of a typical antemortem embolus and its origin in an area of sufficient blood flow.

On a broader basis, the histological dating of the DVT may be summarized as follow [26]:

- During the first week, there are typical Zahn's lines characterizing platelet plug and fibrin deposition in a layered manner on the background of an eroded endothelium in the flowing blood. White blood cells and monocytes with enlarged nuclei are observed.

- During $2^{\text {nd }}$ to $8^{\text {th }}$ weeks, there is endothelial budding and proliferation of fibroblasts. Hemosiderophages as well as fibrinous transformation along with deposition of white cells' nuclear debris and endothelium over the surface of thrombus may be seen.

- From the $8^{\text {th }}$ week, completely hyalinised thrombus with central sinuous cavities and more advanced recanalisation with fresh flowing blood are evident (older than 2 months).

Mansuto, et al. performed the following short-time ranged categorization of thrombus duration, involving evaluations at thromboendothelial junction along with a central progression (using $H \& E$, immunohstochemistry (IHC), immunofluoroscence (IF) and additional staining for collagen/fibrosis) [28].

- Early thrombus ( $\leq 1$ hour): Platelet aggregation with few or no erythrocytes. Presence of factor VIII and fibrinogen. Absence of lymphocyte ( $T$ cell) infiltration.

- Recent thrombus (> $1 \mathrm{~h}$ to $24 \mathrm{~h}$ ): Lymphocytes
( $\mathrm{T}$-cells, CD3+) present. No evidence of fibroblasts and/or fibrosis.

- Recent-medium (24 h to $48 \mathrm{~h}$ ) upto medium (48 $\mathrm{h}$ to $72 \mathrm{~h}$ ) thrombus: Increase in inflammatory cells along with initial degradation with histiocytic and fibroblast cells that showed progressive recruitment. There is lysis of RBCs, WBCs and platelets with prevalent fibrin and cellular debris (homogenisation).

Fibroblast cells with fibrosis are located in peripheral vascular tissue upto third day while they are central and copious from the fourth day onwards.

- Old thrombus (>72 h): Proliferation of endothelial cells, increase of fibrosis and recanalization phenomena.

Because of the pathophysiology and propagation of a thrombus, one may see a broad histological range of thrombosis and organization [7]. Histological examination of residual DVT gives the best opportunity to properly age the thrombus. The embolus typically appears recent and additionally helps in dating the initiating thrombosis.

Thorough sampling of the deep leg veins, with particular attention to the thromboendothelial junction, provides the most information. Endothelial proliferation, collagen deposition, hemosiderin, and neovascularization are common features to note. Because the maximum age of the thrombus usually is most important for medicolegal purposes, adequate and thorough tracing with sampling of the thrombi is needed.

\section{Medicolegal implications}

Pulmonary embolism is the most under-diagnosed condition in most of the nations, frequently being unsuspected as the cause of death by clinicians. It is noteworthy that an originally non-lethal injury may end in death because of venous thrombosis and pulmonary embolism, converting what might be a simple accident or a common assault into a grave legal issue [1].

Victims of trauma-in-general: The victims of many forms of trauma are at risk from pulmonary embolism primarily due to factors such as injury-responsive hypercoagulability of blood, local venous thrombosis in the contused soft tissues and around fractured bones (esp. in the leg and pelvic regions) and the effects of general shock and debility that immobilize and confine the patients to the bed for an invariable period of time.

The various aspects of pulmonary embolism have been investigated in several retrospective autopsy surveys with somewhat different results depending on the era and the source of material $[8,23]$. Some studies have highlighted a complete lack of recognition and deficient autopsy techniques to ascertain pulmonary 
embolism [23]. In cases of trauma and immobility, the peak incidence of PE has been noticed after 1 or 2 weeks $[1,29]$. Most surgical teaching is that a DVT starts "on the table." The use of preoperative, operative, and postoperative prophylaxis usually is dictated by individual patient risk factors and the type and anticipated length of the surgical procedure [2].

Peripartum PTE: Pregnancy is accompanied by unique physiologic changes geared toward accommodating and delivering the growing fetus, followed by recovery and a return to the pregravid state. In most instances, the body is able to adapt to these changes, and the pregnancy from conception through the postpartum period is without major medical complications. However, severe life-threatening pregnancy-related complications do arise that may lead to death of the mother. In the postpartum period/puerperium, the chances of PTE are extremely high during first 6 weeks while in general increase until approximately 12 weeks after delivery $[5,6,30]$.

Sudden maternal death due to PTE may rarely present as a suspicious death. In dealing with such a death, the pathologist should also keep in mind that the woman or her associates might not even have been aware that she was pregnant, as the incidence of DVT in general spans throughout the pregnancy $[12,31]$. As such, the pathologist may be the first doctor to become aware of the pregnancy.

The timing of VTE may have important medicolegal consequences. DVT may form in hours, and the resultant embolism often occurs after a sudden onset of movement after prolonged inactivity.

\section{Conclusion}

Both early diagnosis and treatment of VTE are crucial in order to prevent maternal deaths during the puerperal period. Due to lack of an appropriate and timely diagnosis, undetermined numbers of deaths occur each year because of thromboembolic events. A significant obstructive embolus may prove fatal within a very short period of time. Once a forensic pathologist is involved, a careful autopsy involving through dissection of the leg and pelvic veins along with careful evisceration of organs, evaluation of the probable duration(s) of deep venous thrombi and the pulmonary embolus, the manner of death and a possible exclusion of any medical malpractice must be made. Depending upon the particulars of an investigation, one may offer an opinion of the age and the possible etiology of a thrombus. However, it becomes more difficult to find or establish an initiating link when the interval increases beyond several weeks. Because of the medical, legal, and familial issues, deaths caused by pulmonary emboli need to be properly investigated and documented.

\section{Acknowledgement}

None.

\section{Source of Support/Funding}

Nil.

\section{Statement of Conflicts of Interest}

All the authors declare that they do not have any possible conflicts of interest.

\section{References}

1. Saukko P, Knight B (2016) Knight's forensic pathology. (4 ${ }^{\text {th }}$ edn), Boca Raton, Taylor \& Francis Group, LLC.

2. Dalen JE (2002) Pulmonary embolism: What have we learned since Virchow?: Treatment and prevention. Chest 122: 1801-1817.

3. Becattini C, Agnelli G (2002) Pathogenesis of venous thromboembolism. Curr Opin Pulm Med 8: 360-364.

4. Dolinak D (2005) Pregnancy. In: Dolinak D, Matshes E, Lew E. Forensic pathology: Principles and practice. $\left(1^{\text {st }}\right.$ edn), NY: Elsevier Academic Press, 509-526.

5. Marshall AL (2014) Diagnosis, treatment, and prevention of venous thromboembolism in pregnancy. Postgrad Med 126: $25-34$.

6. Schmidt AP, Szeles TF, Santos WF, Auler JO Jr (2017) Massive pulmonary embolism during the postpartum period: Brief review about diagnosis, prophylaxis and early management. J Obstet Gynaecol 37: 1-4.

7. Gill JR (2005) The medicolegal evaluation of fatal pulmonary thromboembolism. In: Tsokos M, Forensic Pathology Reviews. (1 ${ }^{\text {st }}$ edn), New Jersey: Humana Press, 285-302.

8. Sandler DA, Martin JF (1989) Autopsy proven pulmonary embolism in hospital patients: Are we detecting enough deep vein thrombosis? JR Soc Med 82: 203-205.

9. Lee MY, Kim MY, Han JY, Park JB, Lee KS, et al. (2014) Pregnancy-associated pulmonary embolism during the peripartum period: An 8-year experience at a single center. Obstet Gynecol Sci 57: 260-265.

10. Lucena J, Rico A, Vázquez R, Marín R, Martínez C, et al. (2009) Pulmonary embolism and sudden-unexpected death: prospective study on 2477 forensic autopsies performed at the Institute of Legal Medicine in Seville. J Forensic Leg Med 16: 196-201.

11. Kokturk N, Oguzulgen IK, Demir N, Demirel K, Ekim N (2005) Differences in clinical presentation of pulmonary embolism in older vs. younger patients. Circ J 69: 981-986.

12. Carter N, Rutty GN (2004) The Maternal Death. In: Rutty GN, Essentials of Autopsy Practice: Recent Advances, Topics and Developments. ( $1^{\text {st }}$ edn), London: SpringerVerlag, 73-92.

13. Toglia MR, Weg JG (1996) Venous thromboembolism during pregnancy. N Engl J Med 335: 108-114.

14. Hellgren M, Blombäck M (1981) Studies on blood coagulation and fibrinolysis in pregnancy, during delivery and in the puerperium. I. Normal condition. Gynecol Obstet Invest 12: 141-154.

15. Woodhams BJ, Candotti G, Shaw R, Kernoff PB (1989) Changes in coagulation and fibrinolysis during pregnancy: Evidence of activation of coagulation preceding spontaneous abortion. Thromb Res 55: 99-107.

16. Gerhardt A, Scharf RE, Beckmann MW, Struve S, Bender HG, et al. (2000) Prothrombin and factor $V$ mutations in women with a history of thrombosis during pregnancy and the puerperium. N Engl J Med 342: 374-380. 
17. Rutherford SE, Phelan JP (1986) Thromboembolic disease in pregnancy. Clin Perinatol 13: 719-739.

18. (1986) Prevention of venous thrombosis and pulmonary embolism. NIH consensus development. JAMA 256: 744749.

19. Kakkar VV, Howe CT, Flanc C, Clarke MB (1969) Natural history of postoperative deep-vein thrombosis. Lancet 2 : 230-232.

20. Streiff MB, Agnelli G, Connors JM, Crowther M, Eichinger $S$, et al. (2016) Guidance for the treatment of deep vein thrombosis and pulmonary embolism. J Thromb Thrombolysis 41: 32-67.

21. Wood KE (2002) Major pulmonary embolism: Review of a pathophysiologic approach to the golden hour of hemodynamically significant pulmonary embolism. Chest 121: 877-905.

22. Goldhaber SZ, Elliott CG (2003) Acute pulmonary embolism: Part I: Epidemiology, pathophysiology, and diagnosis. Circulation 108: 2726-2729.

23. Knight B, Zaini MR (1980) Pulmonary embolism and venous thrombosis. A pattern of incidence and predisposing factors over 70 years. Am J Forensic Med Pathol 1: 227-232.

24. Wagenvoort CA (1995) Pathology of pulmonary thromboembolism. Chest 107: 10S-17S.
25. Knight B (1984) The dating of pulmonary emboli. Acta Med Leg Soc (Liege) 34: 190-192.

26. Fineschi V, Turillazzi E, Neri M, Pomara C, Riezzo I (2009) Histological age determination of venous thrombosis: A neglected forensic task in fatal pulmonary thromboembolism. Forensic Sci Int 186: 22-28.

27. Maffeis V, Nicolè L, Rago C, Fassina A (2018) Histological criteria for age determination of fatal venous thromboembolism. Int J Legal Med 132: 775-780.

28. Mansueto G, Costa D, Capasso E, Varavallo F, Brunitto G, et al. (2019) The dating of thrombus organization in cases of pulmonary embolism: An autopsy study. BMC Cardiovasc Disord 19: 250.

29. Lau G (1995) Pulmonary thromboembolism is not uncommon--results and implications of a five-year study of 116 necropsies. Ann Acad Med Singap 24: 356-365.

30. Liu S, Rouleau J, Joseph KS, Sauve R, Liston RM, et al. (2009) Epidemiology of pregnancy-associated venous thromboembolism: A population-based study in Canada. $J$ Obstet Gynaecol Can 31: 611-620.

31. Gherman RB, Goodwin TM, Leung B, Byrne JD, Hethumumi $\mathrm{R}$, et al. (1998) Incidence, clinical characteristics, and timing of objectively diagnosed venous thromboembolism during pregnancy. Prim Care Update Ob Gyns 5: 155-156. 